\title{
Carcinosarcoma of the Palatine Tonsil-Diagnostic and Treatment Approach. Literature Review: Clinical Case
}

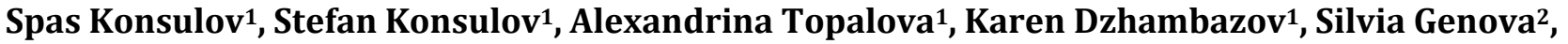 \\ Nikoleta Traykova-Dzhambazova ${ }^{3}$
}

\footnotetext{
${ }^{1}$ Department of ENT Diseases, MU—Plovdiv, University Hospital “Sv. Georgi”, Plovdiv Medical University, Plovdiv, Bulgaria

${ }^{2}$ Department of General and Clinical Pathology, MU—Plovdiv, University Hospital “Sv. Georgi”, Plovdiv Medical University, Plovdiv, Bulgaria

${ }^{3}$ Department of Radiology, MU—Plovdiv, University Hospital “Sv. Georgi”, Plovdiv Medical University, Plovdiv, Bulgaria

Email: skonsulov@abv.bg
}

How to cite this paper: Konsulov, S., Konsulov, S., Topalova, A., Dzhambazov, K., Genova, S. and Traykova-Dzhambazova, N. (2018) Carcinosarcoma of the Palatine Tonsil-Diagnostic and Treatment Approach. Literature Review: Clinical Case. International Journal of Otolaryngology and Head \& Neck Surgery, 7, 20-25.

https://doi.org/10.4236/ijohns.2018.71003

Received: December 2, 2017

Accepted: January 27, 2018

Published: January 30, 2018

Copyright ( 2018 by authors and Scientific Research Publishing Inc. This work is licensed under the Creative Commons Attribution International License (CC BY 4.0).

http://creativecommons.org/licenses/by/4.0/ cc) (i) Open Access

\begin{abstract}
Introduction: Carcinosarcoma of the head and neck is a very rare pathology $<1 \%$. This is a mixed tumor with epithelial and mesenchymal components. It is characterized by high recurrence, metastasis, and mortality rates. Aim: The sporadic nature of carcinosarcomas of the oral cavity, sinonasal tract, larynx, and hypopharynx causes difficulties with the diagnostic and therapeutic approach. Our clinical case is an example of a rare localization of carcinosarcoma, which allows for a detailed examination thereof. Materials and methods: We present a clinical case of a 67-year-old male, admitted to the Otorhinolaryngology Clinic of University Hospital "Sv. Georgi"-Plovdiv, presenting with difficulty swallowing and a lump on the right side of the neck for several months. Computed tomography was performed and a tumor involving the right palatine tonsil was found. The tonsil was surgically removed. The histopathological findings showed a biphasic tumor with epithelial and mesenchymal components-carcinosarcoma. Results: Following monotonsillectomy, the patient was referred to radiotherapy. The local status does not indicate any progression and infiltration into soft tissue. Conclusion: Carcinosarcoma is a malignant mixed tumor with high recurrence, metastasis, and mortality rates. It is difficult to diagnose due to its non-typical location and the need for immunohistochemistry testing, which is not always possible. The therapy for carcinosarcoma of the head and neck is delivered as per protocol for treatment of squamous cell carcinoma due to the absence of a specific one.
\end{abstract}

\section{Keywords}

Carcinosarcoma, Oral Cavity, Tonsil, Mixed Tumor 


\section{Introduction}

Carcinosarcoma is a rare and very aggressive malignancy of the head and neck. It is a special variation of squamous carcinoma comprising epithelial and mesenchymal components, where it is believed that the squamous epithelium has undergone dedifferentiation with sarcomatoid transformation. It has elements of sarcoma and carcinoma, extensively intermixed as to indicate neoplasia of epithelial and mesenchymal tissue. Epithelial component (undifferentiated SCC, squamous cell carcinoma, poorly differentiated adenocarcinoma). It may include: adenoid cystic carcinoma, epithelial myoepithelial carcinoma, salivary duct carcinoma. Non-epithelial component (undifferentiated spindle cell carcinoma). Other reported sarcomatous elements include: fibrosarcoma, osteosarcoma, leiomyosarcoma, liposarcoma, follicular dendritic cell sarcoma, undifferentiated sarcoma, myxoid sarcoma, rhabdomyosarcoma, chondrosarcoma [1]. It has been found to have male predominance, with typical presentation over the age of 60 , and risk factors include a history of smoking, alcohol abuse, poor quality of life, and radiation exposure. The clinical course of this malignancy is not different from that of squamous carcinoma. It presents with non-typical symptoms, sometimes asymptomatically until progression occurs-a metastatic process, usually in the neck region [2]. The therapeutic approach is subject to discussion and it is believed that the treatment protocol for squamous carcinoma is applicable. Occurrence in the oral cavity is extremely rare. Few cases have been reported: tongue, gingiva, palatine tonsil, floor of the mouth, buccal mucosa. Due to the aggressive nature of this tumor and the high rate of recurrence, carcinosarcoma is treated with surgical excision, chemotherapy, radiotherapy.

Aim: The sporadic nature of carcinosarcomas of the oral cavity, sinonasal tract, larynx, and hypopharynx causes difficulties with the diagnostic and therapeutic approach. Our clinical case is an example of a rare localization of carcinosarcoma, which allows for a detailed examination, analyzes and regular follow-up. We intend to keep the data and compare it with the forthcoming cases with this rare type of tumor with respect to complication, metastasis and survival rates.

\section{Materials and Methods}

At the Otorhinolaryngology Clinic of the University Hospital "Sveti Georgi" -Plovdiv, in 2017, one patient was diagnosed with a carcinosarcoma of the right palatine tonsil. He was admitted to this clinic for complaints of a lump, which appeared on the right side of the neck several months earlier, as well as discomfort and pain on swallowing. The patient reported tobacco smoking (20 pack-years). On examination, during mesopharyngoscopy, an exophytic tumor was visualized in the area of the right tonsil, measuring about $3-4 \mathrm{~cm}$ in diameter. Panendoscopy was performed with no signs of secondary tumors or other localization. Paraclinical parameters were normal. At jugular level II, a tumor was found on palpation, suspicious of metastasis, with dimensions of about 4 
$\mathrm{cm}$, which was mobile and painless. Computed tomography was performed, showing a tumor in the area of the right tonsil with non-uniform washout of contrast medium and irregular thickness of the capsule. In the area of the sternocleidomastoid muscle, a round lesion with metastatic characteristics was found (Figure 1).

A biopsy was taken from the major lesion of the right tonsil, and the material was sent for histopathological analysis.

Surgery was performed, excising the tumor through right-sided radical tonsillectomy; the whole tumor was excised, together with the anterior and posterior pillar, up to the base of the tongue on the same side. Right-sided selective neck dissection was performed removing the visible metastasis and lymph nodes at level II and III. The patient was discharged six days after the surgery. The histopathological findings showed a tumor with two malignant components-epithelial from squamous carcinoma, SK AE 1 (AE3/+/positive), and malignant mesenchymal, represented by poorly differentiated mesenchymal chondrosarcoma-Vimentin (+) positive (Figure 2).

The postoperative period was eventless, without any significant complications, infection or postoperative bleeding. Following a review by the Oncology Committee, it was decided that this patient should receive radiation therapy and chemotherapy. The patient attended regular follow-up visits and tests every month. Appointment for PET scan six month after the surgery was arranged.

\section{Discussion}

Carcinosarcoma is a biphasic tumor comprised by squamous and sarcomatoid carcinoma lesions. It was originally described by Virchow in 1894. This type of

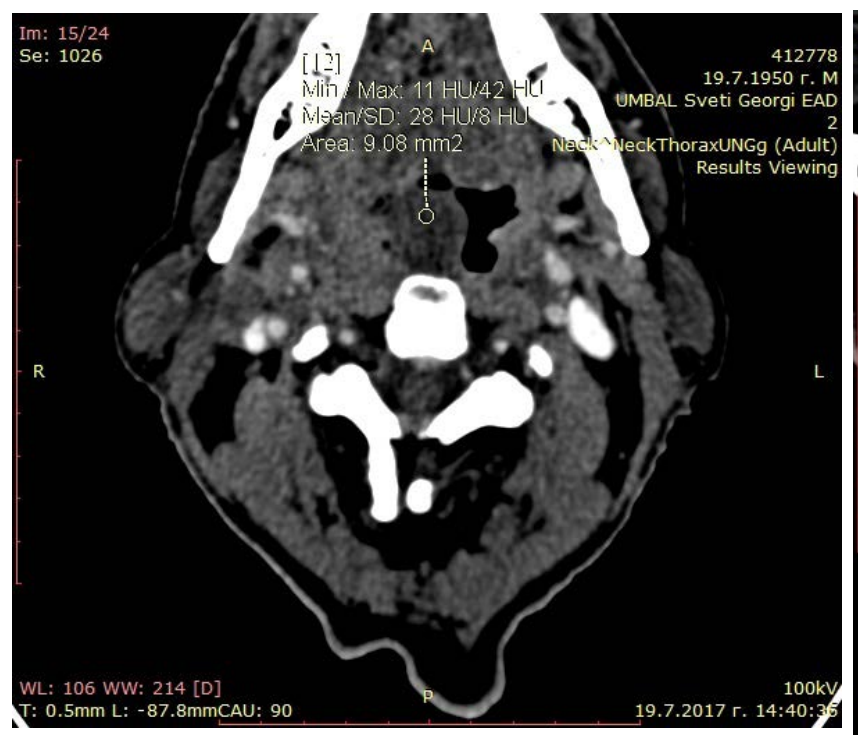

(a)

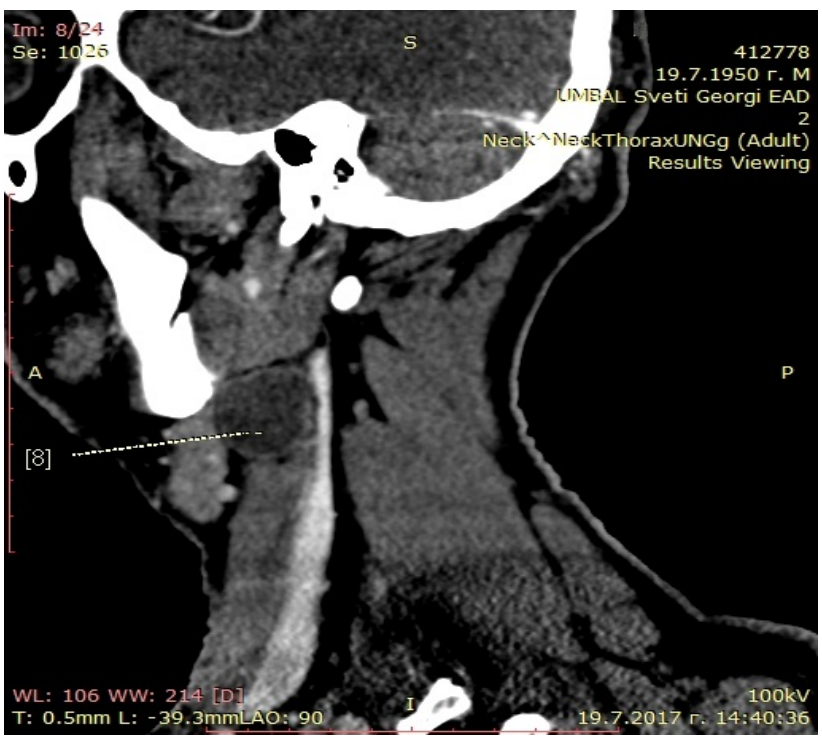

(b)

Figure 1. (a) Computed tomography showing clearly the tumor with non-uniform washout of the contrast medium; (b) The tumor in the neck region has the characteristics of a metastasis. 


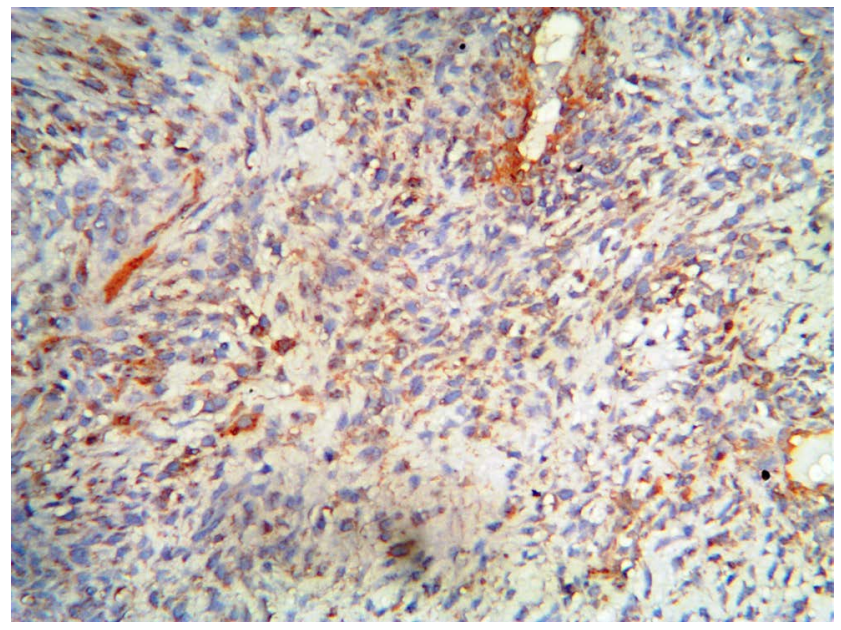

(c)

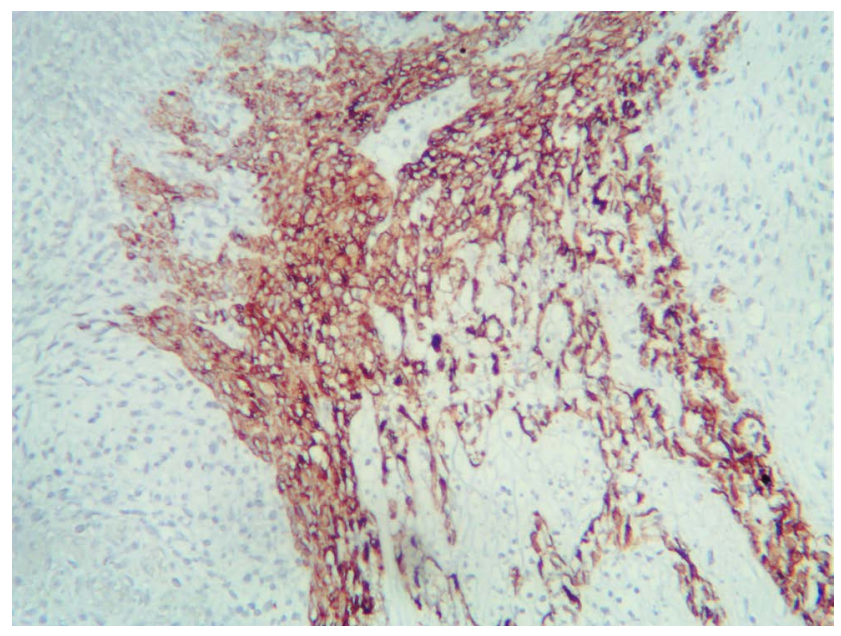

(d)

Figure 2. Histopathological images of the mixed tumor. (a) Mesenchymal Vimetin positive cells, magnification $\times 100$; Positive SK AE1/AE3 in epithelial pockets, magnification $\times 100$.

tumor may arise in any region with squamous epithelium in the human body, both in the respiratory tract and the reproductive organs. Batsakis describes three different categories of mixed tumors:

1) Pleomorphic carcinoma-biphasic epithelial carcinoma. Metastases are uniphasic or mixed.

2) Carcinoma with pseudosarcomatous stroma-epithelial neoplasm with a benign yet atypical stromal reaction. Metastases are epithelial.

3) Carcinosarcoma-heterologous malignancy. Metastases may be uniphasic or mixed [3].

It is rare in the head and neck region; the most frequent sites are the pharynx and the larynx, and extremely rarely-the sinonasal tract [4]. There is a hypothesis about the monoclonal origin of both epithelial and mesenchymal components, which undergo transformation and dedifferentiation. This hypothesis has been supported by immunohistochemistry tests [5].

The most common diagnosis rendered with head and neck fine needle aspiration (FNA) biopsy is metastatic squamous cell carcinoma (SCC). While most cases of metastatic SCC pose little diagnostic difficulty, the diagnosis of metastatic histologic variants of SCC, often coupled with less common etiologies, can be more problematic [6].

Viswanathan S. et al., investigated 103 cases with a confirmed diagnosis of sarcomatoid carcinoma. Age range of the patients was 22 - 90 years (median 53 years), and male:female ratio was 3.7:1. Site distribution was oral cavity $(\mathrm{n}=65$, $63.1 \%)$, larynx (18,17.5\%), oropharynx/hypopharynx (12, 10.7\%), maxilla (6, $5.8 \%)$ and metastatic nodes (2, 1.9\%). A large number of patients (95\%) presented with a mass lesion of less than 1-year duration. Histopathologically, epithelial differentiation was evident on morphology in 48 (46.6\%) cases, only on IHC in $34(33 \%)$ cases, and in $21(20.4 \%)$ cases no epithelial differentiation was found. Typically, tumors were polypoidal $(92,89.3 \%)$ and ulcerated $(95,92.2 \%)$, 
with cells arranged predominantly in fascicles $(59,57.3 \%)$ or in a storiform pattern $(17,16.5 \%)$ [7].

It has been documented that epithelial cells go through a spectrum of progressive phenotypic changes, acquiring a mesenchymal pathway of differentiation metamorphosing to a spindle shape, undergoing a loss of cellular polarity, producing mesenchymal matrix components, and gaining vimentin while losing keratin expression. It has been demonstrated that the phenotypic plasticity of interconversion of epithelium to mesenchyme cells (as seen during embryogenesis) is expressed by a loss of intercellular cohesion, elongation of the cells, loss of basement membrane, production of connective tissue (collagen), and invasion into the stroma [8] [9] [10] [11] [12].

Carcinoma occurs most often in males aged between 60 and 70. The following factors predispose the development of this malignant process-tobacco smoking, alcohol abuse, low social status and quality of life. In our clinical case, tobacco smoking is present as a risk factor. Clinical symptoms vary depending on the location in the head and neck region. The aggressive growth of the carcinosarcoma and its metastatic progression pose challenges to the adequate treatment of this rare type of tumor. Studies by different authors report poor outcomes when radiotherapy is the only treatment modality applied. Therefore, surgical treatment is the primary method of choice. The pre-operative evaluation is of crucial importance for the preparation of the patient. Computed tomography, magnetic resonance tomography and angiography provide information about the size of the tumor, its vascularization, involvement of surrounding soft tissue and metastatic activity in the neck. In view of the rarity of this pathology in global publications, there is limited information about the histopathological variations and the treatment protocol. In our case, the patient underwent radiotherapy after the surgery.

\section{Conclusion}

In conclusion, carcinosarcoma poses challenges for diagnostics and treatment. The presence of both epithelial and mesenchymal components necessitates the use of immunohistopathological analysis and development of a treatment protocol. Surgical treatment is the method of first choice, followed by radiotherapy. The oral cavity is the most frequent site of occurrence, and the inclusion of this type of rare tumor in the differential diagnostic algorithm of malignancies of the pharyngeal mucosa is necessary. Considering the small number of reported cases and the debatable approach to treatment, more detailed geographical studies are suggested as well as studies pertaining to the classification of this type of head and neck tumors.

\section{References}

[1] Anderson, C.E. and Al-Nafussi, A. (2009) Spindle Cell Lesions of the Head and Neck: An Overview and Diagnostic Approach. Diagnostic Histopathology, 15, 264-272. https://doi.org/10.1016/j.mpdhp.2009.02.009 
[2] Stelow, E.B. and Mills, S.E. (2005) Squamous Cell Carcinoma Variants of the Upper Aerodigestive Tract. American Journal of Clinical Pathology, 124, S96-S109. https://doi.org/10.1309/CR5JXUY3J2YGTC1D

[3] Batasakis, J.G. (1981) Pseudosarcoma of the Mucous Membrane in the Head Neck. The Journal of Laryngology \& Otology, 95, 311-316. https://doi.org/10.1017/S0022215100090745

[4] Anonsen, C., Dobie, R.A., Hoekema, D., Huang, T.W. and Gown, A.M. (1985) Carcinosarcoma of the Floor of Mouth. Journal of Otolaryngology, 14, 215-220.

[5] Gupta, R., Singh, S. and Hedau, S. (2007) Spindle Cell Carcinoma of Head and Neck: An Immunohistochemical and Molecular Approach to Its Pathogenesis. Journal of Clinical Pathology, 60, 472-475. https://doi.org/10.1136/jcp.2005.033589

[6] Chute, D.J. and Stelow, E.B. (2010) Cytology of Head and Neck Squamous Cell Carcinoma Variants. Diagnostic Cytopathology, 38, 65-80.

[7] Viswanathan, S., Rahman, K., Pallavi, S., Sachin, J., Patil, A., et al. (2010) Sarcomatoid (Spindle Cell) Carcinoma of the Head and Neck Cosal Region: A Clinicopathologic Review of 103 Cases from a Tertiary Referral Cancer Centre. Head and Neck Pathology, 4, 265-275. https://doi.org/10.1007/s12105-010-0204-4

[8] Thompson, L.D., Wieneke, J.A., Miettinen, M. and Heffner, D.K. (2002) Spindle Cell (Sarcomatoid) Carcinomas of the Larynx: A Clinicopathologic Study of 187 Cases. The American Journal of Surgical Pathology, 26, 153-170.

https://doi.org/10.1097/00000478-200202000-00002

[9] Guarino, M., Tricomi, P., Giordano, F. and Cristofori, E. (1996) Sarcomatoid Carcinomas: Pathological and Histopathogenetic Considerations. Pathology, 28, 298-305. https://doi.org/10.1080/00313029600169224

[10] Nakhleh, R.E., Zarbo, R.J., Ewing, S., et al. (1993) Myogenic Differentiation in Spindle Cell (Sarcomatoid) Carcinomas of the Upper Aerodigestive Tract. Applied Immunohistochemistry, 1, 58-68.

[11] Battifora, H. (1976) Spindle Cell Carcinoma: Ultrastructural Evidence of Squamous Origin and Collagen Production by the Tumor Cells. Cancer, 37, 2275-2282. https://doi.org/10.1002/1097-0142(197605)37:5<2275::AID-CNCR2820370518>3.0. $\mathrm{CO} ; 2-3$

[12] Guarino, M. (1995) Epithelial-to-Mesenchymal Change of Differentiation: from Embryogenetic Mechanism to Pathologic Patterns. Histology and Histopathology, $10,171-184$. 